\title{
3D whole-heart phase sensitive inversion recovery CMR for simultaneous black-blood late gadolinium enhancement and bright-blood coronary CMR angiography
}

Giulia Ginami ${ }^{1 *}$ (D), Radhouene Neji1 ${ }^{1,2}$, Imran Rashid $^{1}$, Amedeo Chiribiri ${ }^{1}$, Tevfik F. Ismail ${ }^{1}$, René M. Botnar ${ }^{1,3}$ and Claudia Prieto ${ }^{1,3}$

\begin{abstract}
Background: Phase sensitive inversion recovery (PSIR) applied to late gadolinium enhancement (LGE) imaging is widely used in clinical practice. However, conventional 2D PSIR LGE sequences provide sub-optimal contrast between scar tissue and blood pool, rendering the detection of subendocardial infarcts and scar segmentation challenging. Furthermore, the acquisition of a low flip angle reference image doubles the acquisition time without providing any additional diagnostic information. The purpose of this study was to develop and test a novel 3D whole-heart PSIR-like framework, named BOOST, enabling simultaneous black-blood LGE assessment and bright-blood visualization of cardiac anatomy.

Methods: The proposed approach alternates the acquisition of a 3D volume preceded by a $T_{2}$-prepared Inversion Recovery ( $T_{2}$ Prep-IR) module (magnitude image) with the acquisition of a $T_{2}$-prepared 3D volume (reference image). The two volumes ( $T_{2}$ Prep-IR BOOST and bright-blood $T_{2}$ Prep BOOST) are combined in a PSIR-like reconstruction to obtain a complementary 3D black-blood volume for LGE assessment (PSIR BOOST). The black-blood PSIR BOOST and the bright-blood $\mathrm{T}_{2}$ Prep BOOST datasets were compared to conventional clinical sequences for scar detection and coronary CMR angiography (CMRA) in 18 patients with a spectrum of cardiovascular disease (CVD).

Results: Datasets from 12 patients were quantitatively analysed. The black-blood PSIR BOOST dataset provided statistically improved contrast to noise ratio (CNR) between blood and scar when compared to a clinical 2D PSIR sequence (15.8 \pm 3.3 and $4.1 \pm 5.6$, respectively). Overall agreement in LGE depiction was found between 3D black-blood PSIR BOOST and clinical 2D PSIR acquisitions, with 11/12 PSIR BOOST datasets considered diagnostic. The bright-blood $\mathrm{T}_{2}$ Prep BOOST dataset provided high quality depiction of the proximal coronary segments, with improvement of visual score when compared to a clinical CMRA sequence. Acquisition time of BOOST ( 10 min), providing information on both LGE uptake and heart anatomy, was comparable to that of a clinical single CMRA sequence.

Conclusions: The feasibility of BOOST for simultaneous black-blood LGE assessment and bright-blood coronary angiography was successfully tested in patients with cardiovascular disease. The framework enables free-breathing multi-contrast whole-heart acquisitions with $100 \%$ scan efficiency and predictable scan time. Complementary information on 3D LGE and heart anatomy are obtained reducing examination time.
\end{abstract}

Keywords: Whole-heart, Black-blood, Bright-blood, Late gadolinium enhancement (LGE), Coronary MR angiography

\footnotetext{
* Correspondence: giulia.ginami@kcl.ac.uk

Dr. Debiao Li served as a guest editor for this manuscript

'School of Biomedical Engineering and Imaging Sciences, King's College

London, St Thomas' Hospital (Lambeth Wing), Westminster Bridge Rd,

London SE1 7EH, UK

Full list of author information is available at the end of the article
} 


\section{Background}

Late gadolinium enhancement (LGE) cardiovascular magnetic resonance (CMR) imaging has become the gold standard for the assessment of myocardial viability in different cardiac pathologies, including myocardial infarction $[1,2]$ and myocarditis [3-5]. In addition, LGE imaging provides pre-interventional assessment of arrhythmogenic substrate in patients undergoing electrophysiology procedures as well as visualization of lesions after ablation [6-8], and is gaining importance in the characterization of fibrosis in non-ischemic cardiomyopathies [9-12]. LGE imaging is typically performed $10-20 \mathrm{~min}$ after the administration of a gadolinium (Gd)-based contrast agent using $\mathrm{T}_{1}$-weighted inversion recovery (IR) sequences [1, 13-15]. The inversion time (TI) is normally set to null the signal from the healthy myocardium, thus enhancing the contrast to noise ratio (CNR) between viable and diseased myocardial tissue. IR sequences, however, are prone to reduced scar to blood and scar to remote myocardium contrast when a sub-optimal TI is selected. Phase-sensitive IR (PSIR) LGE acquisitions have been introduced to provide intrinsic robustness with respect to the TI selection [16]. Conventional PSIR sequences are based on the acquisition of an IR-prepared image (referred to as "magnitude image"), interleaved with a proton density image (referred to as "reference image") that is acquired at a low flip-angle, which are then combined as described in [16]. Although PSIR normally achieves excellent contrast between viable myocardium and scar tissue, the contrast between blood pool and LGE uptake is often suboptimal. This leads to difficulties in delineating sub-endocardial infarcts that are adjacent to the blood pool. Furthermore, unclear borders between scar tissue and blood affect the accuracy of scar segmentation that is crucial for infarct size and transmurality measurements as well as for the planning of electrophysiology procedures [17, 18]. Black-blood PSIR LGE has been introduced [19] to improve the contrast between the blood pool and scar tissue by exploiting an inversion pulse in combination with a $T_{2}$ preparation $\left(\mathrm{T}_{2}\right.$ Prep) module ( $\mathrm{T}_{2}$ Prep-IR) [19-21]. However, a limitation of all PSIR frameworks is that the acquisition efficiency is intrinsically sub-optimal as the low flip-angle reference image has limited diagnostic value. Furthermore, most of the LGE PSIR implementations are limited to $2 \mathrm{D}$ acquisitions that are performed during a breath-hold to minimize respiratory motion artefacts. Recently, free-breathing whole-heart PSIR acquisitions have been introduced [22, 23] and integrated with diaphragmatic navigator gating [24]. The use of diaphragmatic navigator gating, however, leads to reduced scan efficiency and unpredictable acquisition times that can make the selection of the correct TI challenging. In addition, residual imaging artefacts may be observed as a result of the combination of the inversion pulse with the diaphragmatic navigator [25]. In order to overcome these drawbacks, we propose the extension of a 3D whole-heart Bright-blood and black-blOOd phase SensiTive inversion recovery (BOOST) sequence [26] - that has been recently introduced for non-contrast enhanced visualization of coronary lumen and thrombus - to black-blood LGE imaging. The proposed post-contrast BOOST sequence exploits a $T_{2}$ Prep-IR module for the acquisition of the magnitude image, enabling black-blood LGE PSIR reconstruction. Furthermore, the acquisition of the reference image is designed to provide a complementary and fully co-registered bright-blood dataset for the visualization of the heart anatomy, the great vessels, and the coronary lumen. The entire framework has been integrated with image-based navigation [27] to achieve $100 \%$ scan efficiency and predictable scan time. In this study, the feasibility of BOOST for post-contrast simultaneous black-blood LGE imaging and bright-blood heart anatomy, great vessels, and coronary lumen visualization was tested in a cohort of cardiovascular patients at the end of a clinical CMR examination.

\section{Methods}

\section{Framework implementation}

An electrocardiogram -triggered free-breathing 3D whole-heart balanced steady-state free precession (bSSFP) prototype sequence was implemented as described in [26] and as illustrated in Fig. 1. The sequence alternates the acquisition of a $\mathrm{T}_{2}$-prepared IR volume in odd heartbeats ( $\mathrm{T}_{2}$ Prep-IR BOOST, magnitude image) and a $\mathrm{T}_{2}$-prepared volume in even heartbeats (bright-blood $\mathrm{T}_{2}$ Prep BOOST, reference image). Both acquisitions are performed with a Cartesian trajectory with spiral profile order [28] and with a high flip-angle of 90 degrees. A 2D low-resolution image-based navigator (iNAV) [27] is acquired at each heartbeat to estimate translational respiratory motion along the superior-inferior (SI) and right-left (RL) directions and to enable beat-to-beat motion correction with $100 \%$ scan efficiency and predictable scan time. Prior to data acquisition, a rectangular region of interest (ROI) is selected covering the whole heart along the RL direction and covering the base and the mid part of the heart along the SI direction. RL and SI translational motion is then estimated using a template-matching algorithm [29] and motion compensation is performed by modulating the $\mathrm{k}$ space data with a linear shift before image reconstruction [27]. Motion estimation is performed for the $T_{2}$ Prep-IR BOOST and $\mathrm{T}_{2}$ Prep BOOST datasets independently. For each dataset, respiratory motion correction is performed at the end-expiratory level [30].

The motion corrected $\mathrm{T}_{2}$ Prep-IR BOOST and $\mathrm{T}_{2}$ Prep $\mathrm{BOOST}$ volumes are then rigidly co-registered and combined in a PSIR-like reconstruction as described in [16] to obtain a complimentary PSIR BOOST black-blood 


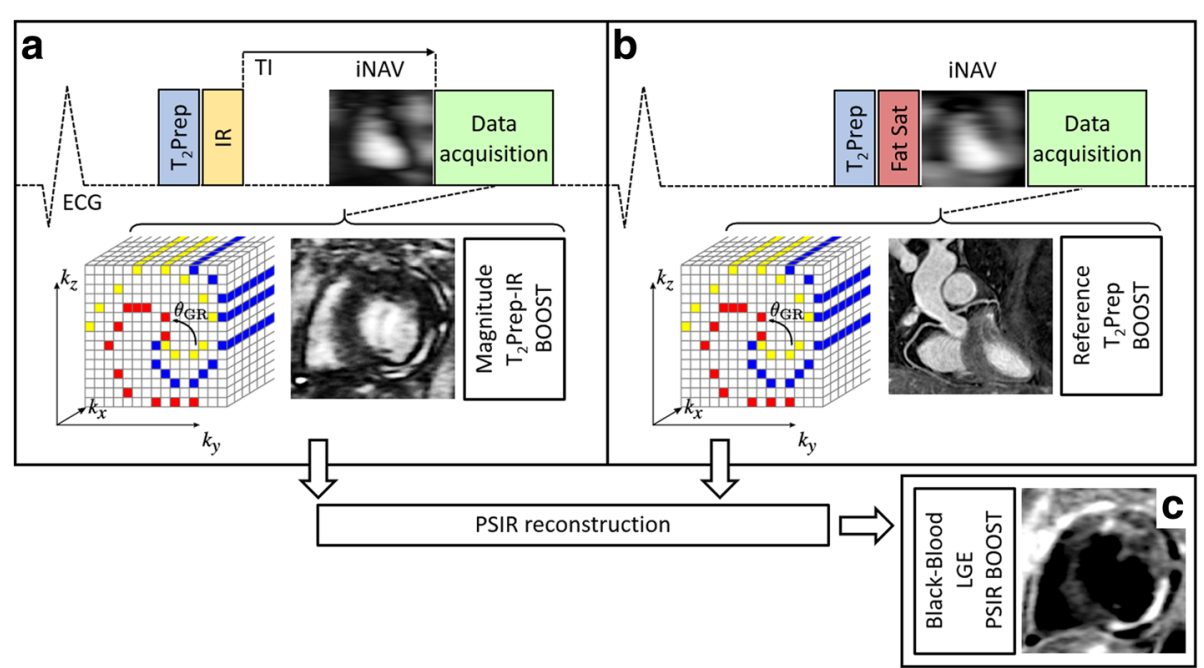

Fig. 1 Proposed post-contrast BOOST framework for simultaneous 3D whole-heart bright-blood coronary angiography and black-blood late gadolinium enhancement (LGE) assessment. $A T_{2}$-prepared inversion recovery ( $T_{2}$ Prep-IR) module is applied at odd heartbeats ( $T_{2}$ Prep-IR BOOST, magnitude image) (a), whereas data acquisition is $T_{2}$ prepared and performed with a high flip angle at even heartbeats (bright-blood $\mathrm{T}_{2}$ Prep-BOOST, reference image) (b). A 3D Cartesian trajectory with spiral profile order [28] is used for data acquisition; data collection is segmented over multiple heartbeats (yellow, red, blue) to minimize the effects of cardiac motion. Even heartbeat acquisitions include a SPIR pulse for fat saturation, while a STIR-like fat suppression is employed in odd heartbeats. 3D data acquisition at each heartbeat is preceded by a low-resolution 2D image-based navigator (iNAV) that is used to estimate translational respiratory motion along the superior-inferior and right-left directions. The two motion corrected datasets (T2Prep-IR BOOST and T2Prep BOOST) are combined in a PSIR-like reconstruction to generate a third, complementary, black-blood dataset (PSIR BOOST) for LGE visualization (c). The motion corrected bright-blood $T_{2}$ Prep BOOST dataset (reference image, b) provides adequate contrast for heart anatomy, great vessel, and coronary lumen visualization

volume. The PSIR-like reconstruction is performed using the $\mathrm{T}_{2}$ Prep BOOST dataset as reference image. As the $\mathrm{T}_{2}$ Prep BOOST is designed for coronary lumen visualization, it exhibits high tissue contrast. Thus, voxel-by-voxel intensity normalization of the resulting PSIR BOOST volume by the reference image is not performed in order to preserve adequate contrast in the resulting black-blood LGE volume.

All acquisitions reported in this study were performed on a 1.5 T CMR system (Magnetom Aera, Siemens Healthineers, Erlangen, Germany) using an 18-channel chest-coil and a 32-channel spine coil. The study was approved by the National Research Ethics Service (15/ NS/0030) and written informed consent was obtained from each participant according to institutional guidelines. Motion estimation and correction, image reconstruction, and PSIR-like computation were implemented using the scanner software (Syngo MR E11A, Siemens Healthineers).

\section{Sequence simulations}

The longitudinal magnetization behavior of healthy viable myocardium, scar and blood was investigated via simulations for the proposed post-contrast BOOST sequence. This was compared against the corresponding simulations for previously published LGE PSIR and bright-blood coronary angiography post-contrast sequences. Sequence simulations were performed in Matlab R2016a (The MathWorks, Inc., Natick, Massachusetts, USA).

Three different CMR sequences were simulated using the extended phase graphs (EPG) formalism [31]: 1) the BOOST sequence as illustrated in Fig. 1; 2) a conventional PSIR sequence for LGE assessment as described in $[16]$; 3) a dedicated $\mathrm{T}_{2}$-prepared bright-blood sequence for coronary CMRA [32]. The simulated tissue parameters were set as follows: healthy viable myocardium $\mathrm{T}_{1}=550 \mathrm{~ms}, \mathrm{~T}_{2}=45 \mathrm{~ms} ; \mathrm{scar} \mathrm{T}_{1}=300 \mathrm{~ms}, \mathrm{~T}_{2}=45 \mathrm{~ms}$; and post-contrast blood $\mathrm{T}_{1}=450 \mathrm{~ms}, \mathrm{~T}_{2}=200 \mathrm{~ms}$. The $\mathrm{T}_{1}$ and $T_{2}$ values of the tissues of interest were set to match those of the standardized phantom [33] used in this study, as described in the following paragraphs, and to approximate the properties of tissues about 15 min after gadolinium contrast injection [33, 34]. Accordingly, imaging parameters were set to match those of the performed phantom acquisitions. For both the BOOST and the conventional PSIR sequences, the TI was set to null the signal from healthy viable myocardium at odd heartbeats (corresponding to $\mathrm{TI}=150 \mathrm{~ms}$ for the BOOST sequence and to $\mathrm{TI}=350 \mathrm{~ms}$ for the conventional PSIR sequence). $\mathrm{T}_{2}$ Prep duration was set to $40 \mathrm{~ms}$ for odd and even heartbeats of the BOOST sequence, and for the CMRA sequence. A flip-angle of 90 degrees was simulated for both the acquisition of the magnitude and the bright-blood reference 
image in the BOOST sequence as well as for the acquisition of the magnitude image in the conventional PSIR sequence. A flip-angle of 8 degrees was simulated for the reference image of the conventional PSIR sequence. For the CMRA sequence, a 90 degrees flip angle was simulated for each heartbeat. For all three sequences (BOOST, conventional PSIR, and CMRA), and for each individual heartbeat, a data acquisition duration equal to $120 \mathrm{~ms}$ was simulated, corresponding to $33 \mathrm{k}$-space lines. To minimize signal oscillations during acquisition, and to generate the iNAVs at each individual heartbeat, 14 bSSFP linear rampup pulses were simulated prior to imaging data acquisition. The heart-rate was simulated at 60 beats per minute with a total of 50 heartbeats (of which 2 were dummy heartbeats).

For all the simulated sequences, expected magnetization $\mathrm{M}_{\mathrm{z}} / \mathrm{M}_{0}$ as well as absolute signal differences between the tissues of interest were computed. All the absolute signal differences (that are preserved in the final PSIR reconstruction) were computed at the beginning of the data acquisition using centric k-space ordering.

\section{Phantom experiments Data acquisition}

A standardized $T_{1}$ and $T_{2}$ phantom, with different vials resembling $T_{1}$ and $T_{2}$ values of the most relevant cardiac compartments [33], was used for data acquisition. Healthy myocardium, scar, and post-contrast blood $\mathrm{T}_{1}$ and $\mathrm{T}_{2}$ values were identical to the simulated ones. Data acquisition was performed using the BOOST sequence, the conventional PSIR sequence, and the dedicated CMRA acquisition. $\mathrm{T}_{2}$ Prep durations, TIs, heart rate, number of k-space lines acquired per heartbeat and flip angle values were kept identical to those used in the simulations. Additional imaging parameters included: transverse orientation, Field of view $(\mathrm{FOV})=$ $320 \times 320 \times 60 \mathrm{~mm}^{3}$, in-plane spatial resolution $=1 \mathrm{~mm}^{2}$, slice thickness $=2 \mathrm{~mm}$, echo-time $(\mathrm{TE}) /$ repetition time $(\mathrm{TR})=1.56 / 3.6 \mathrm{~ms}$, pixel bandwidth $977 \mathrm{~Hz} /$ pixel. The PSIR BOOST reconstruction was performed with and without intensity normalization for comparison purposes.

\section{Data analysis}

Signal to noise ratio (SNR) and CNR were quantified for the three sequences (BOOST, conventional PSIR, and dedicated CMRA). SNR of blood ( $\left.\mathrm{SNR}_{\text {blood }}\right)$, healthy viable myocardium $\left(\mathrm{SNR}_{\mathrm{myo}}\right)$ and scar $\left(\mathrm{SNR}_{\mathrm{scar}}\right)$ were calculated for the odd heartbeats of BOOST and conventional PSIR, together with CNR between blood and healthy myocardium ( $\left.\mathrm{CNR}_{\text {blood-myo }}\right)$, scar and blood $\left(\mathrm{CNR}_{\text {scar-blood }}\right)$, and scar and healthy viable myocardium $\left(\mathrm{CNR}_{\text {scar-myo }}\right) . \mathrm{SNR}_{\text {blood }}$ and $\mathrm{CNR}_{\text {blood-myo }}$ were quantified for even heartbeats for both the BOOST and the conventional PSIR sequence as well as for the dedicated CMRA acquisition. For the PSIR images obtained from the BOOST sequence (with and without intensity normalization) and the conventional PSIR sequence, $\mathrm{CNR}_{\text {blood-myo, }}, \mathrm{CNR}_{\text {scar-blood, }}$ and $\mathrm{CNR}_{\text {scar-myo }}$ were quantified after the removal of low spatial frequency signal components as described in [16, 35].

\section{In-vivo experiments \\ Data acquisition}

Eighteen patients $(52.7 \pm 13.2$ years, 9 males $)$ who were referred for a clinical CMR examination were recruited for this study. In 16 out of 18 (89\%) patients, a conventional 2D multi-slice and multi breath-hold bSSFP Cartesian PSIR sequence [16] was acquired in different orientations (four chamber view, three chamber view, short-axis view) starting $10 \mathrm{~min}$ after gadobutrol (0.2 mmol $/ \mathrm{kg}$ ) administration (Gadovist, Bayer, Berlin, Germany). Relevant imaging parameters for this acquisition include: FOV $=292 \times 152 \mathrm{~mm}^{2}$, slice thickness $=$ $8 \mathrm{~mm}$, in plane spatial resolution $=1.4 \mathrm{~mm}^{2}, 10$ slices acquired for the short axis view, TE/TR $=1.26 / 2.9 \mathrm{~ms}$, pixel bandwidth $=775 \mathrm{~Hz} /$ pixel, flip angle $=45$ degrees, ECG triggering to the most quiescent diastolic period. The TI (typically ranging from $200 \mathrm{~ms}-300 \mathrm{~ms}$ ) was selected with a dedicated TI scout scan and was set to null the signal from the healthy viable myocardium. In 7 out of 18 (39\%) subjects, a conventional free-breathing navigator-gated bright-blood whole-heart $\mathrm{T}_{2}$-prepared bSSFP (CMRA) Cartesian sequence was acquired after the breath-hold 2D PSIR sequences. For this acquisition, imaging parameters were set as follows: sagittal orientation, subjectspecific $F O V=410 \times 307 \times 160-192 \mathrm{~mm}^{3}$, in plane spatial resolution $=1.4 \mathrm{~mm}^{2}$, slice thickness $=1.4 \mathrm{~mm}$, TE $/ \mathrm{TR}=$ $1.56 / 3.6 \mathrm{~ms}$, pixel bandwidth $=575 \mathrm{~Hz} /$ pixel, flip angle $=$ 90 degrees, $\mathrm{T}_{2}$ Prep duration $=40 \mathrm{~ms}, 2 \times$ GRAPPA parallel imaging acceleration [36] with 24 calibration lines. Respiratory motion was compensated using diaphragmatic navigator gating and tracking (tracking factor equal to 0.6 [37]), with an acceptance window placed in endexpiration and with an amplitude equal to $\pm 3.5 \mathrm{~mm}$.

At the end of the clinical examination, the proposed ECG-triggered BOOST sequence was acquired under free-breathing and using the following imaging parameters: coronal orientation, in-plane spatial resolution $=1 \mathrm{~mm}^{2}$, slice thickness $=4 \mathrm{~mm}$ (interpolated to $2 \mathrm{~mm}$ during image reconstruction), subject-specific $\mathrm{FOV}=320 \times 320 \times 80$ $130 \mathrm{~mm}^{3}, \mathrm{TE} / \mathrm{TR}=1.56 / 3.6 \mathrm{~ms}$, pixel bandwidth $977 \mathrm{~Hz} /$ pixel. For both the magnitude image $\mathrm{T}_{2}$ Prep-IR BOOST and the reference image $\mathrm{T}_{2}$ Prep BOOST, the flip angle was set to 90 degrees and the $\mathrm{T}_{2}$ Prep duration was equal to $40 \mathrm{~ms}$. The subject-specific TI (typically ranging in the interval 100-180 ms) was selected to null the signal from viable healthy myocardium by acquiring a dedicated $2 \mathrm{D}$ BOOST TI scout scan during a breath-hold; such scout 
scan consisted of a magnetization-prepared cine sequence where a $T_{2}$ Prep-IR module is applied right after the Rwave at odd heartbeats, whereas $T_{2}$-preparation solely is performed at the beginning of the cardiac cycle in even heartbeats. Cine frames belonging to the odd heartbeats were visually inspected to determine the optimized TI. In odd heartbeats, fat signal was suppressed for the effect of the inversion pulse (STIR-like approach) [38], while spectral pre-saturation (SPIR) [39] was used to suppress signal from epicardial fat at even heartbeats (Fig. 1). Images obtained in patients for whom BOOST data acquisition did not start later than $40 \mathrm{~min}$ after contrast injection were considered for further quantitative and qualitative data analyses. This temporal restriction was set to avoid a too pronounced washout of the contrast agent.

\section{Quantitative data analysis}

Quantitative data analysis was performed for the images acquired with the clinical 2D PSIR sequence, and for the 3D black-blood LGE and bright-blood CMRA datasets obtained with BOOST (PSIR BOOST and $\mathrm{T}_{2}$ Prep BOOST, respectively). ROIs were manually drawn in blood, healthy viable myocardium and scar tissue (when present) at matching anatomical locations for both the clinical 2D PSIR and the 3D whole-heart PSIR BOOST images. Background noise was computed from a ROI with uniform signal positioned at the level of the liver, following the removal of low spatial frequency signal components as performed for the phantom images $[16,35]$. CNR $\mathrm{CNlood}-\mathrm{myo}_{\text {, }} \mathrm{CNR}_{\text {scar-blood }}$ and $\mathrm{CNR}_{\text {scar-myo }}$ were quantified for both the clinical 2D PSIR and the 3D whole-heart PSIR BOOST images. CNR values were quantified in the subjects for whom both the $2 \mathrm{D}$ PSIR and BOOST sequences were acquired, and compared using a paired 2-tailed Student t-test. $P=0.05$ was set as the threshold to determine statistical significance. Acquisition times of all the acquired sequences (2D PSIR acquisition - including pauses between breath-holds -, clinical whole-heart CMRA, and BOOST) were recorded. In addition, scan efficiency was recorded for the clinical CMRA acquisition with diaphragmatic navigator gating.

\section{Qualitative data analysis}

All the datasets (3D black-blood PSIR BOOST, 3D bright-blood $\mathrm{T}_{2}$ Prep BOOST, conventional 3D CMRA acquisition, and clinical 2D PSIR) were anonymized and stored in a randomized order. Qualitative grading of the anonymized images was performed by two experienced cardiologists (T.F.I. and I.R., SCMR III certification) blinded to clinical data. For all the LGE images (blackblood 3D PSIR BOOST and 2D clinical PSIR), presence and location of LGE were assessed. Furthermore, images were graded in terms of diagnostic quality on consensus basis using a 4-point scale system where 1 indicates a fully diagnostic dataset without the presence of artefacts, 2 indicates a diagnostic dataset with only minor artefacts present, 3 indicates a diagnostic dataset with significant artefacts, and 4 indicates an artefacts-rendering images non-diagnostic dataset. Subjective scores for LGE visualization were compared with a paired Wilcoxon signed-rank test to assess statistical differences; $P<0.05$ was considered statistically significant. Statistical analyses were performed considering the cases for whom both the clinical 2D PSIR and the BOOST sequences were acquired. For all acquired bright-blood datasets (conventional 3D CMRA and $\mathrm{T}_{2}$ Prep BOOST), the ability to identify the origin and the proximal course of the coronary arteries was graded for four relevant coronary segments: left main (LM), left anterior descending coronary artery (LAD), left circumflex coronary artery (LCX), and right coronary artery (RCA). Grading was performed on consensus basis using a 4-point scale system as that used for the grading of LGE datasets.

\section{Results}

All data acquisitions and reconstructions were carried out successfully and all quantified endpoints are reported hereafter.

\section{Sequence simulations}

All simulated pulse sequences (proposed post-contrast BOOST sequence, conventional PSIR sequence [16], and dedicated post-contrast CMRA acquisition [32]) and the resulting steady state magnetization behavior for blood, myocardium and scar tissues are reported in Fig. 2. For the $\mathrm{T}_{2}$ Prep-IR BOOST sequence (odd heartbeats) the expected magnetization $M_{z} / M_{0}$ of the healthy viable myocardium, blood and scar varied within the intervals $0.000-0.059,-0.158-0.003$, and $0.108-0.116$, respectively, during imaging data acquisition. This resulted in an absolute signal difference between myocardium and scar of +0.108 and of +0.266 between blood and scar. For the conventional PSIR sequence, and in correspondence to the acquisition of the magnitude image, the expected magnetization $M_{z} / M_{0}$ of the healthy viable myocardium varied within the interval $-0.002-0.058$, whereas the expected magnetization $\mathrm{M}_{\mathrm{z}} / \mathrm{M}_{0}$ of blood and scar varied within the intervals $0.056-0.146$ and $0.207-0.144$, respectively. This resulted in an absolute signal difference between healthy myocardium and scar amounting to +0.208 , and of +0.151 between blood and scar. For the conventional PSIR sequence, the reference image at even heartbeats (low flip-angle) exhibited high values of expected magnetization $\mathrm{M}_{\mathrm{z}} / \mathrm{M}_{0}$, varying in the intervals $0.807-0.858,0.877-0.919$ and $0.951-0.967$, for healthy viable myocardium, blood, and scar tissue, respectively. Conversely, even heartbeats of the BOOST 


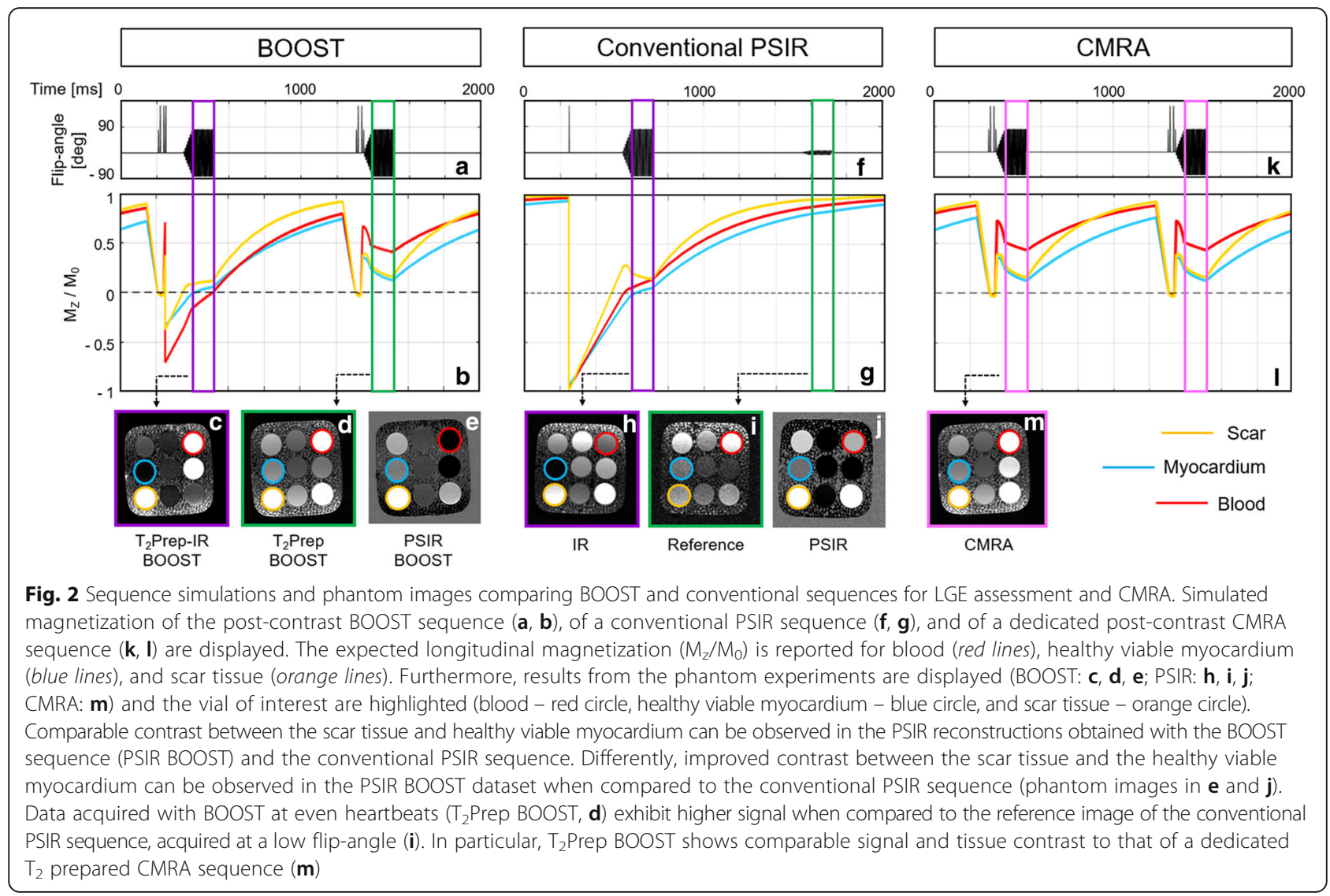

sequence $\left(\mathrm{T}_{2}\right.$ Prep BOOST) exhibited reduced expected magnetization $M_{z} / M_{0}$ for the tissues of interest, since data acquisition is performed with higher flip-angle and is preceded by a $T_{2}$ Prep. Specifically, the expected magnetization $\mathrm{M}_{\mathrm{z}} / \mathrm{M}_{0}$ of healthy viable myocardium, blood and scar varied within the intervals $0.230-0.123$, $0.490-0.420$ and $0.267-0.167$, respectively. This resulted in a blood/myocardium ratio of 2.13 , which is adequate for anatomy and coronary lumen visualization. Similarly, for the conventional post-contrast CMRA sequence, the expected magnetization $\mathrm{M}_{\mathrm{z}} / \mathrm{M}_{0}$ of healthy viable myocardium, blood and scar varied within the intervals $0.220-0.100,0.498-0.438$, and $0.267-0.161$, respectively, leading to a blood/myocardium ratio equal to 2.26 .

\section{Phantom experiments}

Phantom images obtained with the proposed post-contrast BOOST sequence, the conventional PSIR sequence [16], and the dedicated post-contrast CMRA acquisition are shown in Fig. 2. ROIs corresponding to post-contrast blood, healthy viable myocardium, and scar are indicated by red, blue and yellow circles, respectively. All the endpoints that were quantified for the phantom acquisitions are summarized in the Additional file 1 . The $\mathrm{T}_{2}$ Prep-IR BOOST phantom dataset showed strong signal from both blood and scar tissue, while providing suppression of the signal belonging to the vial mimicking the viable myocardium. The magnitude image of the conventional PSIR acquisition showed effective suppression of signal from the viable myocardium, and high signal from the vial mimicking the scar. The reference image $\mathrm{T}_{2}$ Prep BOOST, designed for the visualization of the heart anatomy, great vessels, and coronary lumen, showed $\mathrm{SNR}_{\text {blood }}$ and $\mathrm{CNR}_{\text {blood-myo }}$ comparable to those provided by the dedicated CMRA acquisition. Conversely, the reference image of the conventional PSIR acquisition (acquired at a low flip angle) showed reduced $\mathrm{SNR}_{\text {blood }}$ and $\mathrm{CNR}_{\text {blood-myo }}$ The PSIR reconstructions obtained using the BOOST sequence with and without normalization are displayed in Fig. 3. Reduced tissue contrast is observed for the PSIR reconstruction with intensity normalization. Conversely, tissue contrast was restored in the PSIR reconstruction without intensity normalization. The PSIR BOOST phantom dataset (obtained without intensity normalization) showed effective blood signal suppression, leading to improved $\mathrm{CNR}_{\text {scar-blood }}$ when compared to the more conventional PSIR sequence.

\section{In-vivo experiments}

Quantitative and qualitative data analysis was performed for 12 of 18 patients for whom BOOST data acquisition started less than $40 \mathrm{~min}$ after contrast agent injection. The average time after injection for the BOOST datasets 


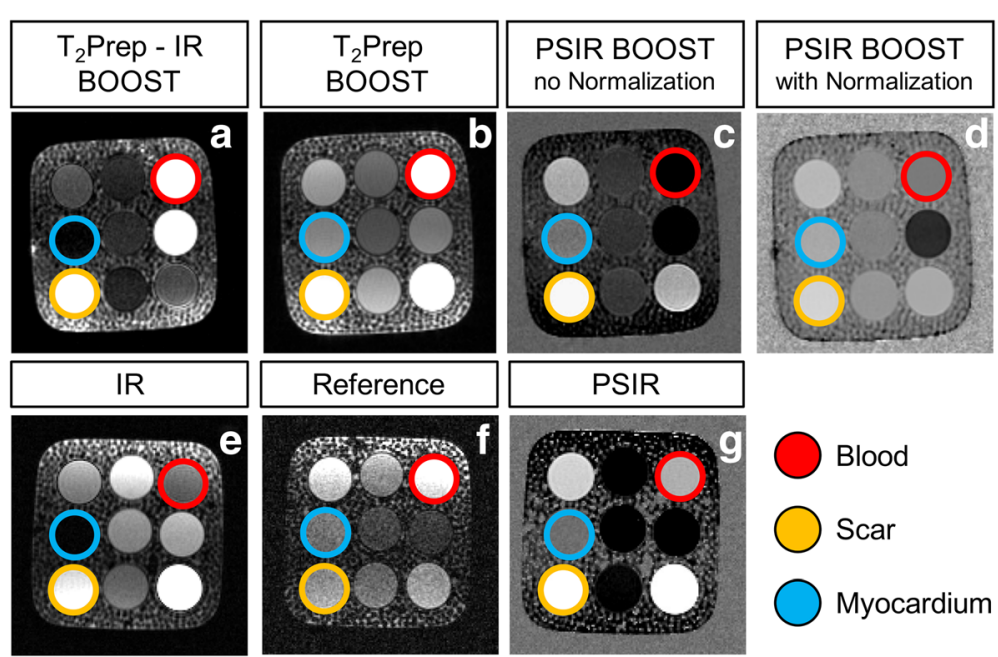

Fig. 3 Phantom images obtained with the BOOST and the conventional PSIR sequence. Imaging data were acquired by nulling the signal from the healthy viable myocardium (blue vial) in the magnitude images $(\mathbf{a}, \mathbf{e})$. Differently from $\mathbf{f}$, the $T_{2}$ Prep BOOST dataset, acquired at a high flipangle, exhibits both high signal from the blood (red vial) and pronounced contrast between blood and healthy viable myocardium (b). The PSIR reconstruction obtained with BOOST and using intensity normalization (d) shows reduced tissue contrast, which is restored once intensity normalization is not applied (c). Furthermore, such restored contrast between the scar tissue (orange vial) and the healthy viable myocardium is comparable to that of the PSIR reconstruction in $(\mathbf{g})$, while improved contrast between scar and blood can be appreciated

was 27:47 $\pm 3: 35 \mathrm{~min}$. Among those patients, the acquisition of the clinical 2D PSIR sequence and of the clinical 3D whole-heart CMRA with diaphragmatic navigator was performed in 10 and 6 cases, respectively. Clinical, imaging, and demographic characteristics are summarized in Table 1. Acquisition times were 7:48 $\pm 4: 03 \mathrm{~min}$ for the clinical 2D PSIR sequence (including pauses between breath-holds), 13:06 \pm 3:05 $\mathrm{min}$ for the conventional CMRA acquisition with diaphragmatic navigator (with an average scan efficiency of $\sim 47 \%$ ), and 12:07 \pm 1:56 $\mathrm{min}$ for data acquisition with BOOST that provides both blackblood LGE and bright-blood anatomical images. For the BOOST sequence, image-based navigation enabled data acquisition with $100 \%$ scan efficiency (i.e., none of the acquired data was discarded during image reconstruction) and predictable scan time. Furthermore, translational motion correction led to effective respiratory motion correction for both the bright-blood $\mathrm{T}_{2}$ Prep and the black-blood PSIR BOOST datasets in most cases, as shown in Fig. 4 for two representative patients.

\section{Quantitative data analysis}

The endpoints quantified for the conventional 2D PSIR sequence amounted to $\mathrm{CNR}_{\text {blood-myo }}=15.2 \pm 8.1$, $\mathrm{CNR}_{\text {blood-scar }}=4.1 \pm 5.6$ and $\mathrm{CNR}_{\text {scar-myo }}=12.3 \pm 9.3$. The black-blood PSIR BOOST datasets presented effective nulling of the blood signal, leading to significantly

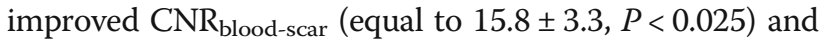
significantly reduced $\mathrm{CNR}_{\text {blood-myo }}(4.2 \pm 3.6, P<0.001)$ when compared to the clinical 2D PSIR sequence (Fig. 5).
Quantified $\mathrm{CNR}_{\text {scar-myo }}$ was equal to $13.02 \pm 4.56$ ( $\mathrm{P}=\mathrm{NS}$ in comparison to the clinical 2D PSIR sequence).

\section{Qualitative data analysis}

Eleven of 12 3D PSIR BOOST datasets were considered diagnostic, with an average grade of $1.75 \pm 1.21$; specifically, $8 / 12$ cases were graded 1 , one single case was graded 2, two cases - where incomplete blood suppression was observed - were graded 3, and one single case was graded 4 due to the presence of residual motion and pronounced artefacts originating from the rigid translation of the chest wall and arms. Complete correspondence between clinical 2D PSIR acquisitions and 3D PSIR BOOST datasets was found in $8 / 10$ cases in terms of LGE findings and location of the LGE uptake. In two individual cases (Patient 08 and Patient 10) LGE was not visible in the 3D PSIR BOOST dataset. Both datasets were graded 1 , meaning no residual artefacts were visible and optimal blood signal suppression was achieved. In these two cases, however, BOOST data acquisition started 39:57 min and 39:32 min after contrast injection, following a conventional 3D CMRA acquisition (duration 17:04 $\mathrm{min}$ and 11:31 $\mathrm{min}$ ) that was performed between the 2D clinical PSIR and the 3D BOOST sequences. This particularly pronounced delay between the two LGE acquisitions, together with the absence of residual artefacts and the achievement of adequate blood signal suppression in the PSIR BOOST datasets, suggests that contrast agent washout prevented adequate scar depiction. Clinical 2D PSIR images were graded 1 for all the 10 patients where the sequence was acquired. No 
Table 1 Summary of patients' data used for quantitative analysis

\begin{tabular}{|c|c|c|c|c|c|c|}
\hline & Gender & Age (years) & $\begin{array}{l}\text { Heartbeats } \\
\text { per minute }\end{array}$ & Clinical Condition & LGE findings & $\begin{array}{l}\text { 3D whole heart } \\
\text { CMRA }\end{array}$ \\
\hline Patient 01 & $\mathrm{~F}$ & 25 & 85 & Atrial fibrillation & No & Yes \\
\hline Patient 02 & $\mathrm{~F}$ & 30 & 70 & $\begin{array}{l}\text { Atrial fibrillation } \\
\text { (previous myocarditis) }\end{array}$ & No & No \\
\hline Patient 03 & $\mathrm{~F}$ & 48 & 70 & $\begin{array}{l}\text { Eosinophilic Granulomatous Polyangitis } \\
\text { (Churg-Strauss syndrome) }\end{array}$ & No & No \\
\hline Patient 04 & M & 50 & 80 & Myocarditis & $\begin{array}{l}\text { Yes } \\
\text { Diffuse mid-wall }\end{array}$ & No \\
\hline Patient 05 & M & 67 & 55 & Myocardial infarction & $\begin{array}{l}\text { Yes } \\
\text { Transmural }\end{array}$ & No \\
\hline Patient 06 & M & 56 & 65 & $\begin{array}{l}\text { Advanced Hypertensive } \\
\text { heart disease }\end{array}$ & $\begin{array}{l}\text { No } \\
\text { (no 2D PSIR) }\end{array}$ & Yes \\
\hline Patient 07 & M & 66 & 70 & Myocardial infarction & $\begin{array}{l}\text { Yes } \\
\text { Transmural and subendocardial }\end{array}$ & No \\
\hline Patient 08 & M & 62 & 80 & Myocardial infarction & $\begin{array}{l}\text { Yes } \\
\text { Mid-wall }\end{array}$ & Yes \\
\hline Patient 09 & M & 59 & 45 & Myocardial infarction & $\begin{array}{l}\text { Yes } \\
\text { Subendocardial }\end{array}$ & Yes \\
\hline Patient 10 & $\mathrm{~F}$ & 30 & 75 & Myocardial infarction & $\begin{array}{l}\text { Yes } \\
\text { Subendocardial }\end{array}$ & Yes \\
\hline Patient 11 & $\mathrm{~F}$ & 74 & 80 & Myocardial infarction & $\begin{array}{l}\text { Yes } \\
\text { Transmural }\end{array}$ & No \\
\hline Patient 12 & M & 52 & 75 & Suspected myocardial infarction & $\begin{array}{l}\text { No } \\
\text { (no 2D PSIR) }\end{array}$ & Yes \\
\hline
\end{tabular}

Furthermore, the presence of LGE findings is stated ("no 2D PSIR" pertains the cases where 2D PSIR acquisition was not performed and presence of LGE uptake was assessed with BOOST only). In addition, it is indicated whether the acquisition of the conventional, 3D whole-heart CMRA sequence was performed or not

statistically significant difference was found in terms of visual grading $(\mathrm{P}=\mathrm{NS})$ when comparing the 3D PSIR BOOST datasets and the clinical 2D PSIR counterpart (average grade of BOOST in these 10 patients: $1.80 \pm$ 1.31). In terms of coronary conspicuity, image quality scores evaluated by consensus grading were $1.50 \pm 1.22$ (LM), $2.00 \pm 1.54$ (LAD), $2.50 \pm 1.64$ (LCX) and $2.83 \pm 1.16$ (RCA) for the conventional CMRA with diaphragmatic navigator (6 patients, Table 1). A trend of improvement in terms of coronary delineation was quantified with BOOST in the same 6 patients; for those subjects, LM and LAD were graded 1 in all cases. For LCX and RCA, average grades were equal to $1.50 \pm 1.22$ and $1.50 \pm 0.83$, respectively. A visual comparison between $\mathrm{T}_{2}$ Prep BOOST datasets and conventional CMRA is shown in Fig. 6. Overall visual grading obtained with $\mathrm{T}_{2}$ Prep BOOST (considering the entire cohort of 12 patients, which includes cases where the acquisition of the conventional CMRA was not performed) was $1.07 \pm 0.27$ for the $L M, 1.07 \pm 0.27$ for the LAD, $1.07 \pm 0.27$ for the LCX, and $1.53 \pm 0.77$ for the RCA.

Fusion of a bright-blood $\mathrm{T}_{2}$ Prep BOOST with a blackblood PSIR BOOST datasets (obtained with the Horos software, V1.1.7) is illustrated in Fig. 7 to demonstrate the location and transmurality of the infarct obtained from the black-blood PSIR dataset.

\section{Discussion}

In this study, we extended the use of a novel PSIR-like framework, referred to as BOOST, to post-contrast applications for simultaneous 1) black-blood LGE assessment and 2) bright-blood heart anatomy, great vessels, and coronary lumen visualization. With the BOOST framework, the acquisition of the magnitude image $\left(\mathrm{T}_{2}\right.$ Prep-IR BOOST) is based on a $\mathrm{T}_{2}$ Prep-IR module for optimal contrast between the blood pool and scar tissue after PSIR computation (black-blood PSIR BOOST). Furthermore, the acquisition of the reference image (bright-blood $\mathrm{T}_{2}$ Prep BOOST) is performed with a high flip-angle and it is preceded by a $\mathrm{T}_{2}$ Prep module. This ensures adequate signal and tissue contrast for the visualization of heart anatomy, great vessels, and the coronary lumen. In contrast to previously published approaches providing a single bright-blood dataset for the simultaneous visualization of LGE and proximal coronary arteries [40], our framework generates two separate yet co-registered 3D volumes, each one being specifically designed and optimized for the visualization of the coronary lumen (bright-blood $\mathrm{T}_{2}$ Prep BOOST) and myocardial scar (black-blood PSIR BOOST).

Sequence simulations and phantom acquisitions showed that the proposed post-contrast PSIR BOOST dataset achieves improved scar-blood contrast when compared to 


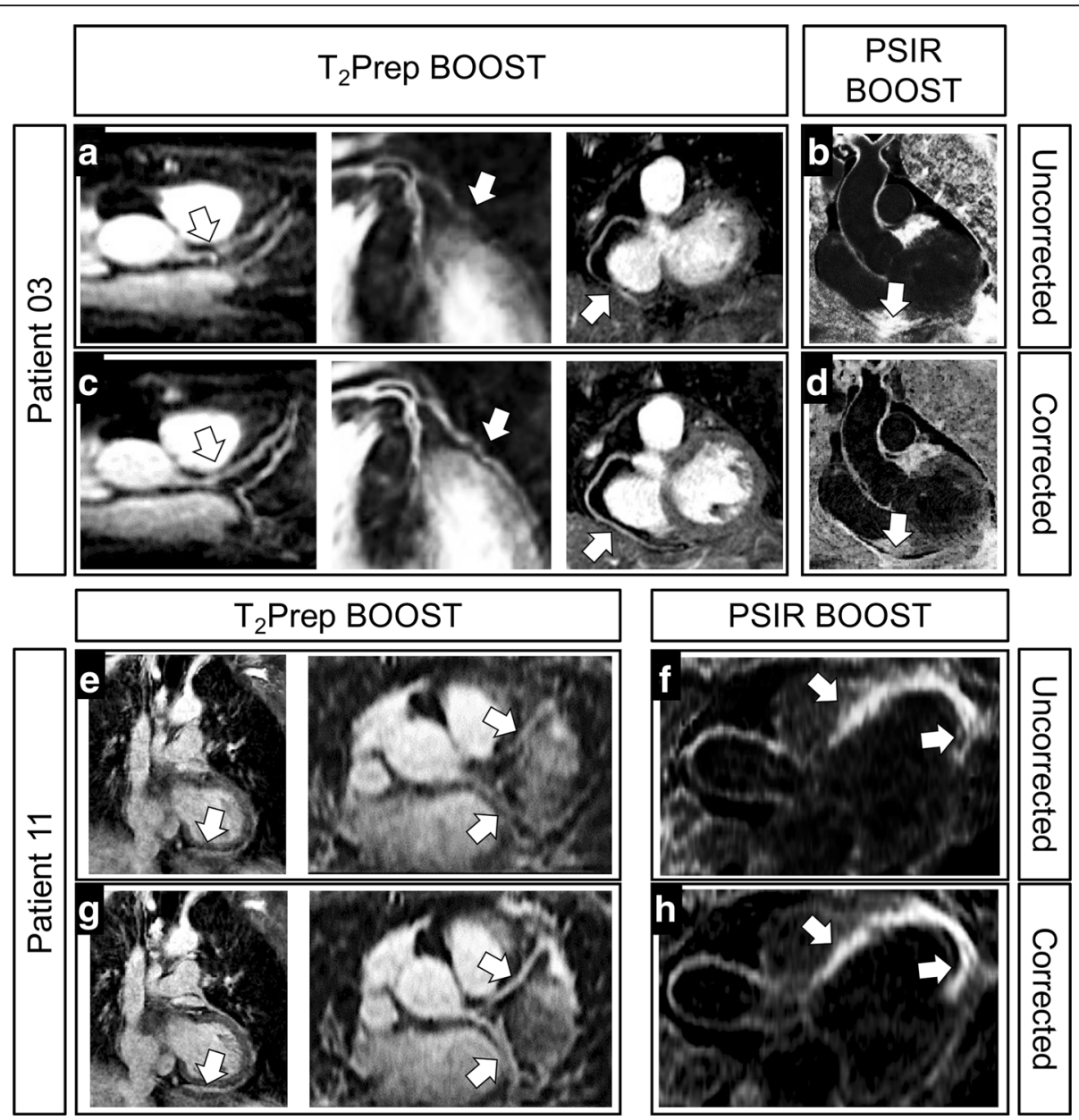

Fig. 4 Improvement in BOOST image quality after translational motion correction in two representative patients. The use of translational motion correction along the SI and RL directions reduces blurring artefacts and improves coronary vessel sharpness in the bright-blood $\mathrm{T}_{2}$ Prep BOOST datasets (arrows in $\mathbf{a}, \mathbf{c}, \mathbf{e}$, and $\mathbf{g}$ ). Motion compensation recovers also small details as showed in the zoomed images. Furthermore, improved image sharpness can be observed on the black-blood PSIR-like reconstructions (arrows in $\mathbf{b}, \mathbf{d}, \mathbf{f}$, and $\mathbf{h}$ ), where a sharper delineation of the LGE uptake can be appreciated following motion correction ( $\mathbf{f}$ versus $\mathbf{h}$ )

a more conventional PSIR sequence for LGE imaging [16]; this was confirmed by in vivo measurements in patients. While the PSIR BOOST volume provided adequate LGE depiction in most of the patients with positive findings, phantom experiments indicate higher $\mathrm{CNR}_{\text {scar-myo }}$ in the $\mathrm{T}_{2}$ Prep-IR BOOST datasets, where precise viable myocardial nulling is achieved; this can be qualitatively appreciated in vivo as shown in Fig. 5. As such, referring to the $\mathrm{T}_{2}$ Prep-IR BOOST dataset for the detection of subtle, non-ischemic, fibrosis patterns might be preferable; this aspect, however, needs further investigation and will be analyzed in future studies. Furthermore, sequence simulations show that the bright-blood $\mathrm{T}_{2}$ Prep BOOST dataset provides $\mathrm{SNR}_{\text {blood }}$ and $\mathrm{CNR}_{\text {blood-myo }}$ similar to those of a dedicated $\mathrm{T}_{2}$-prepared post-contrast CMRA acquisition. In vivo acquisitions showed that respiratory motion corrected bright-blood $\mathrm{T}_{2}$ Prep BOOST datasets allowed visualization of the origin and the proximal course of the coronary arteries (LM, LAD, LCX, and RCA) with high diagnostic quality. A trend of improvement was observed in comparison to the conventional CMRA; respiratory motion compensation performed with diaphragmatic navigator gating assumes a fixed linear correlation between the respiratory motion of the liver and that of the heart. The fixed correlation factor of 0.6 [37] that was used in this study might have been inexact for some of the subjects, thus leading to sub-optimal motion compensation. Conversely, with the use of image-based navigation, respiratory motion information can be directly extracted from the heart itself, thus avoiding the risk of imprecise approximations. In addition, with image-based navigation, it is possible to correct for movements along both SI and $\mathrm{RL}$ directions [27]. These aspects may have been a contributing factor of the improved coronary delineation that was obtained with BOOST. Furthermore, and as predicted by sequence simulations, the black-blood PSIR BOOST 


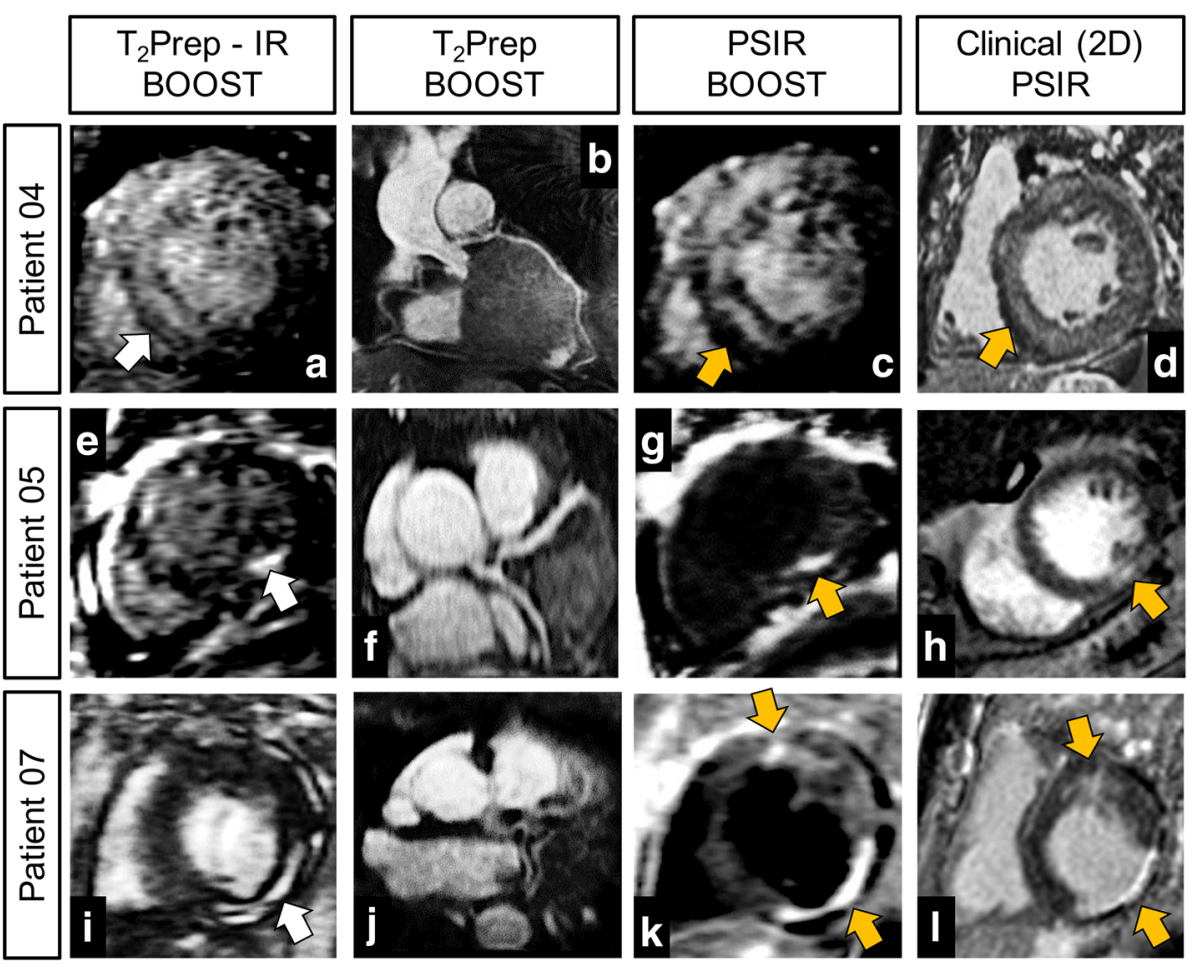

Fig. 5 Comparison between the proposed 3D whole-heart BOOST framework and the clinical 2D PSIR acquisition. Images in a, e, and i show the LGE uptake as depicted in the T2Prep-IR BOOST datasets (white arrows), where signal from the blood pool is present and the viable myocardium is suppressed. Reformats in $\mathbf{b}, \mathbf{f}$, and $\mathbf{j}$ show the coronary reformats obtained from the $3 \mathrm{D}$ whole-heart bright-blood $\mathrm{T}_{2}$ Prep BOOST dataset. Complementary 3D black-blood LGE images obtained with BOOST are shown in $\mathbf{c}, \mathbf{g}$, and $\mathbf{k}$. All the images from the T 2 Prep-IR BOOST and the PSIR BOOST datasets were reformatted to match the orientation of the clinical 2D PSIR acquisitions ( $\mathbf{d}, \mathbf{h}$, and $\mathbf{I})$. The LGE uptake identified in both the $T_{2}$ Prep-IR BOOST and PSIR BOOST datasets matches that of the clinical 2D PSIR acquisition. Furthermore, improved contrast between the scar tissue and the blood pool can be appreciated in the 3D PSIR BOOST datasets when compared to the 2D PSIR acquisition ( $\mathbf{g}, \mathbf{k}$ versus $\mathbf{h}, \mathbf{I}$, orange arrows). LGE uptake appears more shallow and blood pool signal is not entirely suppressed in Patient 04 with myocarditis (c, $\mathbf{d}$, orange arrows), due to a longer $\mathrm{Tl}$

reconstruction provided visualization of LGE with diagnostic quality in most cases and significantly improved $\mathrm{CNR}_{\text {scar-blood }}$ was quantified in comparison to clinical 2D PSIR acquisitions [16].

The PSIR reconstruction performed with the proposed framework exactly follows that described in [16], with the exception of the intensity normalization step that is conventionally performed at the end of the PSIR pipeline. In contrast to previously published post-contrast PSIR sequences [16], the reference image $\left(T_{2}\right.$ Prep BOOST) acquired in our approach exhibits high tissue contrast, thus preventing the application of surface coil intensity normalization. In fact, the presence of high tissue contrast in the reference image significantly alters the resulting contrast of the normalized PSIR reconstruction (Fig. 3). The use of surface coil intensity normalization is typically exploited to compensate for large variations in the intensity of the image caused by rapid fall-off of the surface-coil fields, thus improving the local tissue contrast. This was shown to be particularly beneficial for the visualization of subendocardial infarcts, given the fact that the contrast between scar tissue and blood is particularly reduced in conventional PSIR acquisitions [16]. With this new sequence configuration, however, intrinsically enhanced contrast between blood and scar tissue is provided using a $\mathrm{T}_{2}$ Prep-IR module for the acquisition of the magnitude image; in addition, the use of pre-scan based normalization readily available on commercial scanners can be exploited to compensate for variations in signal intensity. This might alleviate the need for surface coil intensity normalization, however further validation may be needed to corroborate this point.

The integration of the framework with image-based navigation enabled data acquisition during freebreathing with $100 \%$ scan efficiency and predictable scan time. The acquisition time for BOOST (approximately $12 \mathrm{~min}$ ) was similar to that of a conventional CMRA acquisition with diaphragmatic navigator (approximately $13 \mathrm{~min}$ ), considering an average scan efficiency of $50 \%$ and $2 \times$ parallel imaging acceleration. The BOOST framework, however, provides both a bright- and black- 


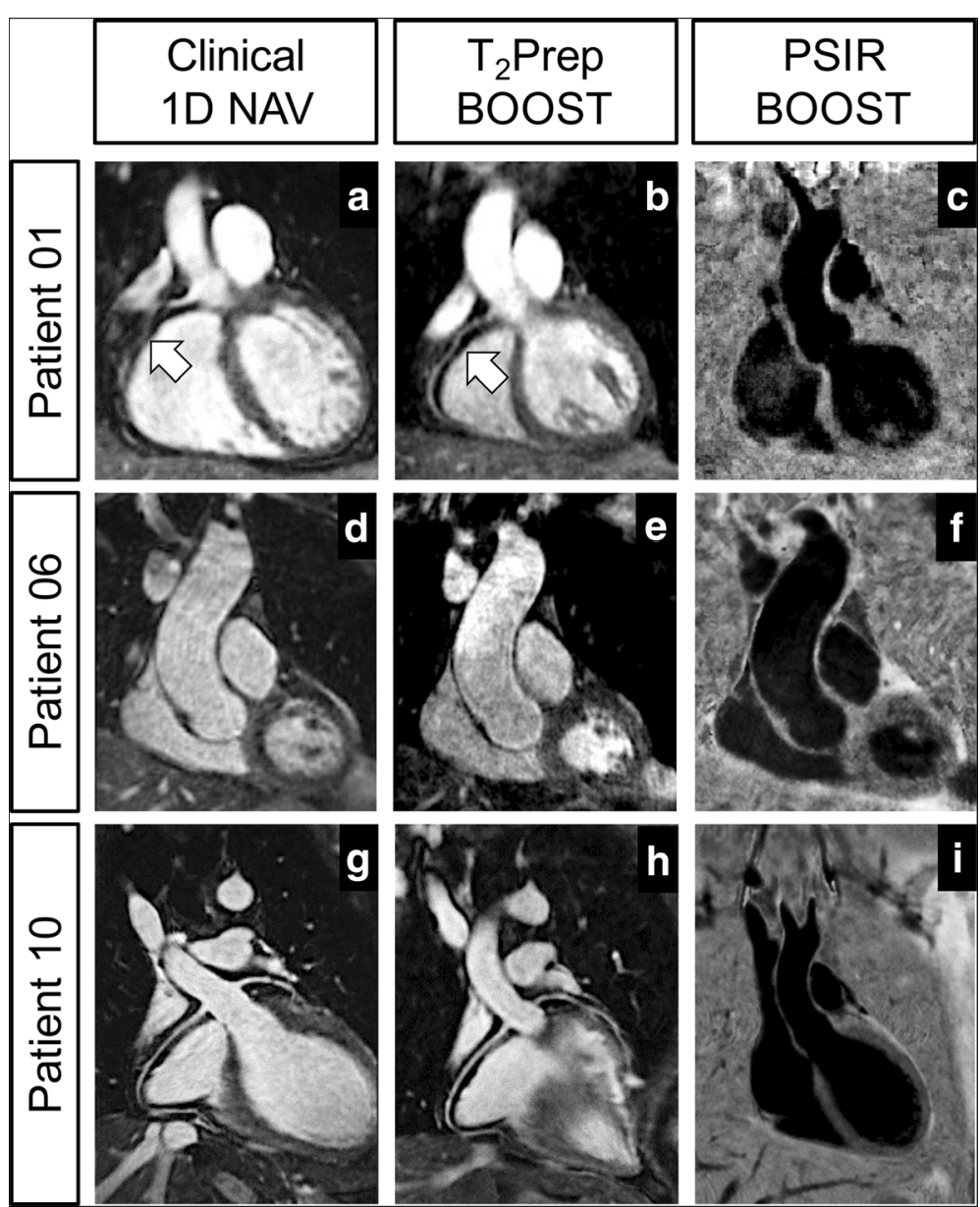

Fig. 6 Comparison between conventional 3D whole-heart acquisition with diaphragmatic navigator and the proposed bright-blood $T_{2}$ Prep BOOST. Improved delineation of the RCA can be appreciated in Patient 01 with $\mathrm{T}_{2}$ Prep BOOST when compared to the conventional CMRA acquisition (arrows in $\mathbf{a}, \mathbf{b}$ ). Dilated aorta can be observed in Patient 06 due to the presence of hypertensive heart disease (d, e). Excellent coronary delineation was obtained with both sequences in Patient $10(\mathbf{g}, \mathbf{h})$. Furthermore, the complementary black-blood PSIR BOOST datasets for LGE assessment are shown in $\mathbf{c}, \mathbf{f}$, and $\mathbf{i}$

blood dataset in the same acquisition time, whereas the overall acquisition of conventional CMRA and 2D PSIR sequences was about $20 \mathrm{~min}$ in our cohort of patients. This intrinsic efficiency of the BOOST framework holds potentials for reducing the scan time that is currently needed to perform a complete CMR examination. This might be particularly beneficial in the case of claustrophobic, anxious, or clinically unstable patients. Additionally, reducing the overall examination time would imply economic benefits and reduction of patients waiting lists. Future technical developments of the BOOST sequence will include the integration of acceleration techniques $[36,41,42]$ to improve both the nominal acquisition time as well as the spatial resolution. Furthermore, improvements in the acquired spatial resolution might enable isotropic acquisitions that would, for instance, allow for more robust visualization of the mid and distal coronary arteries. Similarly, the achievement of higher spatial resolution could benefit tissue characterization, allowing for a more accurate delineation of scar tissue and enabling a more accurate image fusion between the bright-blood $\mathrm{T}_{2}$ Prep BOOST and the black-blood PSIR BOOST datasets for the assessment of scar location and transmurality. Additionally, the framework will be integrated with algorithms for arrhythmia rejection that could further improve the image quality that was obtained in this study. Currently, BOOST is combined with image-based navigation enabling in-line translational motion correction along the SI and RL directions. However, the breathing pattern in patients is often more complex and involves translation, rotation, and non-rigid deformations [43-45]. Therefore, future technical developments will aim at combining the BOOST framework with strategies for non-rigid respiratory motion correction [46] that might be particularly beneficial in very sick patients who often have irregular 

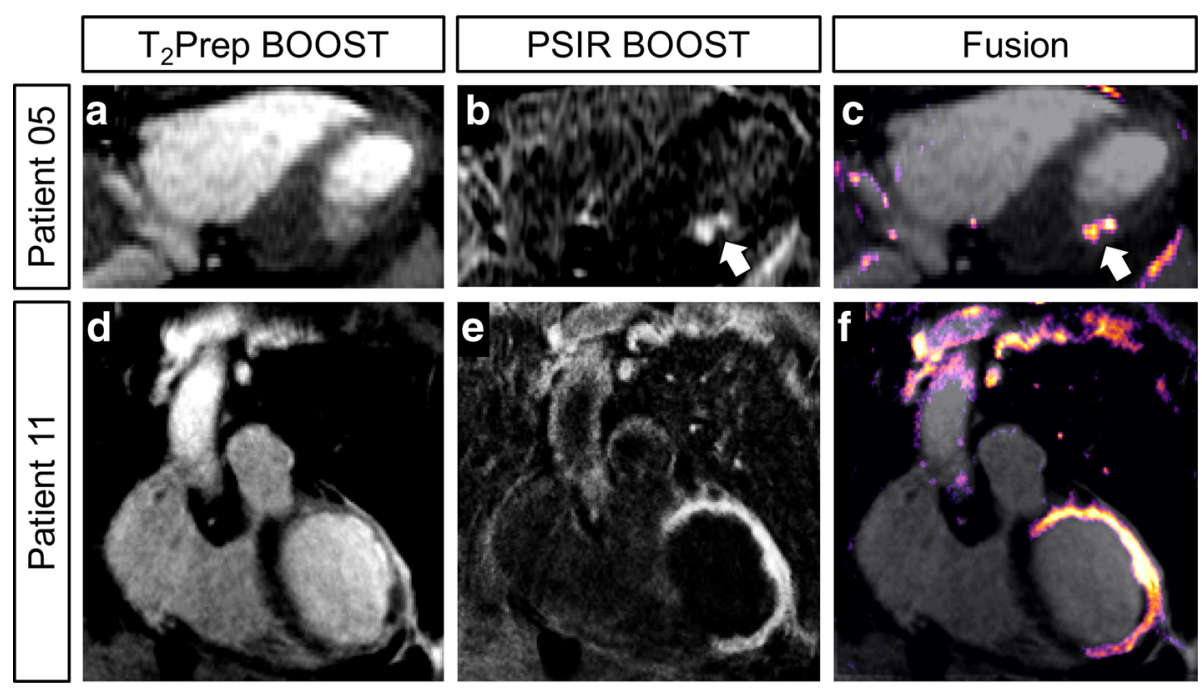

Fig. 7 Fusion of the bright-blood $T_{2}$ Prep BOOST and the black blood PSIR BOOST datasets. Images correspond to two representative patients with positive LGE findings. Bright-blood images for visualization of the heart anatomy are shown in $\mathbf{a}, \mathbf{d}\left(\mathbf{T}_{2}\right.$ Prep-BOOST). Complementary visualization of scar tissue (PSIR BOOST) is shown in $\mathbf{b}$, e; these datasets could potentially be used for an easy scar segmentation, as unclear border between the surrounding tissues and the scar itself have disappeared. Fusion images, where the anatomical localization of the scar can be retrieved, are shown in $\mathbf{c}, \mathbf{f}$

breathing patterns [47]. In addition, the use of non-rigid respiratory motion correction may help to reduce ghosting artefacts that may originate from rigid translation of static tissues such as the chest wall and arms during the motion correction process. Similarly, a rigid registration between the $T_{2}$ Prep-IR BOOST dataset and the $T_{2}$ Prep BOOST dataset is currently performed prior PSIR computation to compensate for residual mis-registration errors; this may be also sub-optimal and the use of nonrigid registration could further improve the quality of the resulting PSIR BOOST dataset and additionally reduce the risk of phase errors that may originate in portions of the image where phases are not varying smoothly (e.g. in correspondence to the interface between different tissues).

In this study, the BOOST acquisition was performed at the end of a clinical CMR examination as it was considered unethical to potentially jeopardize the acquisition of conventional LGE data at the expense of a novel sequence at this stage. Injection timing was optimized to provide optimal contrast agent concentration during the acquisition of the clinical 2D PSIR sequences, thus providing suboptimal contrast conditions for the BOOST scan. This was noticed particularly in two specific cases (Patient 08 and Patient 10), where LGE uptake could not be depicted despite the absence of motion artefacts and the achievement of optimal blood signal suppression. Furthermore, as the BOOST acquisition was performed at the end of the clinical scan, there may have been more respiratory or heart-rate irregularities that might have had an additional detrimental effect on the image quality that was obtained with BOOST. However, scan time was not prolonged by more than $15 \mathrm{~min}$ at most. Therefore, future studies are warranted to rigorously compare the proposed post-contrast 3D BOOST sequence and conventional 2D PSIR acquisitions by, for instance, randomizing the order of the two acquisitions and by performing separate $\mathrm{Gd}$ injections to ensure equivalent contrast conditions. Kellman et al. [19] demonstrated that black-blood LGE provides improved conspicuity of subendocardial infarcts; future studies will aim at investigating the accuracy of black-blood PSIR BOOST for the quantification of scar transmurality and, thus, regional viability assessment. Furthermore, accuracy in the detection and quantification of ischaemic scar will be validated. Similarly, further clinical validation of BOOST is needed in comparison to conventional CMRA in patients with angiographically confirmed coronary artery disease.

The improved contrast between blood pool and scar provided by the proposed black-blood PSIR BOOST images may facilitate scar segmentation. However, the nulling of the blood and viable myocardium signal reduces the depiction of the heart anatomy and might challenge the localization of the scar itself. This challenge can be addressed by fusing the co-registered black blood PSIR dataset with the bright blood whole heart dataset, which then allows both scar and myocardial anatomy visualization as shown in Fig. 7. This characteristic makes this framework particularly suitable for the planning of electrophysiology procedures. Similarly, the framework may be beneficial for the visualization of lesions after ablation and follow-up of patients. This further enlarges the spectrum of potential clinical applications of post-contrast 
BOOST, that will be tested in dedicated studies in the upcoming future.

\section{Conclusions}

We demonstrated the feasibility of simultaneous blackblood LGE imaging and bright-blood visualization of cardiac anatomy, the great vessels, and the coronary artery lumen using a novel motion corrected multi-contrast 3D imaging sequence, referred to as BOOST. Data acquisition with BOOST was performed in free-breathing and ensures whole-heart coverage, 100\% scan efficiency, and predictable scan time. The framework was validated in a group of cardiac patients and showed high quality depiction of the coronary arteries in comparison to standard CMRA and good agreement with 2D PSIR LGE scar visualization. This novel sequence has a broad spectrum of potential clinical applications.

\section{Additional file}

Additional file 1: Table summarizing the quantified endpoints for all the performed phantom acquisitions. (DOCX $14 \mathrm{~kb}$ )

\begin{abstract}
Abbreviations
BOOST: Bright-blood and black-blood phase sensitive inversion recovery; bSSFP: Balanced steady state free precession; CMR: Cardiovascular magnetic resonance; CMRA: Coronary magnetic resonance angiography; CNR: Contrast to noise; EPG: Extended phase graph (simulation); FOV: Field of view; Gd: Gadolinium; GRAPPA: Generalized autocalibrating partially parallel acquisition; iNAV: Image-based navigator; IR: Inversion recovery; LAD: Left anterior descending coronary artery; LCX: Left circumflex coronary artery:; LGE: Late gadolinium enhancement; LM: Left main coronary artery; PSIR: Phase-sensitive inversion recovery; RCA: Right coronary artery; ROI: Region of interest; SNR: Signal to noise; $T_{2}$ Prep: $T_{2}$-prepared; $T_{2}$ Prep-IR: $\mathrm{T}_{2}$-prepared inversion recovery $\backslash$ TE: Echo time; TI: Inversion time; TR: Repetition time
\end{abstract}

\section{Acknowledgements}

The views expressed are those of the authors and not necessarily those of the National Health Service, the National Institute for Health Research, or the Department of Health.

\section{Funding}

This work was supported by the following grants: 1) EPSRC EP/N009258/1, EP/ P001009/1, EP/P007619/1, MRC MR/L009676/1, and FONDECYT N $\left.{ }^{\circ} 1161051,2\right)$ the Wellcome EPSRC Centre for Medical Engineering at King's College London (WT 203148/Z/16/Z) and 3) the Department of Health via the National Institute for Health Research (NIHR) comprehensive Biomedical Research Centre award to Guy's \& St Thomas' NHS Foundation Trust in partnership with King's College London and King's College Hospital NHS Foundation Trust.

\section{Availability of data and materials}

Anonymized dicom images from patient acquisitions are not publicly available but are available from the corresponding author upon reasonable request.

\section{Authors' contributions}

$\mathrm{GG}, \mathrm{RN}, \mathrm{RMB}$, and CP designed the study. GG and RN implemented the acquisition and reconstruction framework on the scanner software. GG performed sequence simulations and phantom experiments. GG, IR, and TFI performed the data analysis. All the authors contributed to the acquisition of clinical research data. All the authors participated in drafting and revising the manuscript, and read and approved its final version.

\section{Ethics approval and consent to participate}

The study was approved by the National Research Ethics Service and written informed consent was obtained for all the subjects. Anonymized data were analyzed at the School of Biomedical Engineering and Imaging Sciences (King's College London) at the St. Thomas' Hospital.

\section{Consent for publication}

All the subjects provided written informed consent for the publication of accompanying images in this manuscript. The consent forms are held in the patients' clinical notes and are available to the Editor-in-Chief upon request.

\section{Competing interests}

R.N. is employed by Siemens Healthcare Limited. All the other Authors declare that they do not have competing interests.

\section{Publisher's Note}

Springer Nature remains neutral with regard to jurisdictional claims in published maps and institutional affiliations.

\section{Author details}

${ }^{1}$ School of Biomedical Engineering and Imaging Sciences, King's College London, St Thomas' Hospital (Lambeth Wing), Westminster Bridge Rd, London SE1 7EH, UK. ${ }^{2}$ MR Research Collaborations, Siemens Healthcare Limited, Sir William Siemens Square Frimley, Camberley GU16 8QD, UK. ${ }^{3}$ Escuela de Ingeniería, Pontificia Universidad Católica de Chile, Vicuna Mackenna, 4860 Santiago, Chile.

Received: 23 August 2017 Accepted: 6 November 2017 Published online: 27 November 2017

\section{References}

1. Kim RJ, Fieno DS, Parrish TB, Harris K, Chen EL, Simonetti O, Bundy J, Finn JP, Klocke FJ, Judd RM. Relationship of MRI delayed contrast enhancement to irreversible injury, infarct age, and contractile function. Circulation. 1999; 100:1992-2002.

2. Fieno DS, Kim RJ, Chen EL, Lomasney JW, Klocke FJ, Judd RM. Contrastenhanced magnetic resonance imaging of myocardium at risk: distinction between reversible and irreversible injury throughout infarct healing. J Am Coll Cardiol. 2000;36:1985-91.

3. Friedrich MG, Strohm O, Schulz-Menger J, Marciniak H, Luft FC, Dietz R. Contrast media-enhanced magnetic resonance imaging visualizes myocardial changes in the course of viral myocarditis. Circulation. 1998;97:1802-9.

4. Abdel-Aty $H$, Boye $P$, Zagrosek A, Wassmuth $R$, Kumar A, Messroghli D, Bock P, Dietz R, Friedrich MG, Schulz-Menger J. Diagnostic performance of cardiovascular magnetic resonance in patients with suspected acute myocarditis: comparison of different approaches. J Am Coll Cardiol. 2005; 45:1815-22.

5. Friedrich MG, Sechtem U, Schulz-Menger J, Holmvang G, Alakija P, Cooper LT, White JA, Abdel-Aty H, Gutberlet M, Prasad S, et al. Cardiovascular magnetic resonance in myocarditis: a JACC white paper. J Am Coll Cardiol. 2009:53:1475-87.

6. Lardo AC, McVeigh ER, Jumrussirikul P, Berger RD, Calkins H, Lima J, Halperin HR. Visualization and temporal/spatial characterization of cardiac radiofrequency ablation lesions using magnetic resonance imaging. Circulation. 2000;102:698-705.

7. Dickfeld T, Kato R, Zviman M, Lai S, Meininger G, Lardo AC, Roguin A, Blumke D, Berger R, Calkins H, Halperin H. Characterization of radiofrequency ablation lesions with gadolinium-enhanced cardiovascular magnetic resonance imaging. J Am Coll Cardiol. 2006;47:370-8.

8. Vergara GR, Marrouche NF. Tailored management of atrial fibrillation using a LGE-MRI based model: from the clinic to the electrophysiology laboratory. J Cardiovasc Electrophysiol. 2011;22:481-7.

9. Hunold P, Schlosser T, Vogt FM, Eggebrecht H, Schmermund A, Bruder O, Schuler WO, Barkhausen J. Myocardial late enhancement in contrast-enhanced cardiac MRI: distinction between infarction scar and non-infarction-related disease. AJR Am J Roentgenol. 2005;184:1420-6.

10. Bohl S, Wassmuth $R$, Abdel-Aty H, Rudolph A, Messroghli D, Dietz R, SchulzMenger J. Delayed enhancement cardiac magnetic resonance imaging reveals typical patterns of myocardial injury in patients with various forms of non-ischemic heart disease. Int J Cardiovasc Imaging. 2008;24:597-607. 
11. Ismail TF, Prasad SK, Pennell DJ. Prognostic importance of late gadolinium enhancement cardiovascular magnetic resonance in cardiomyopathy. Heart. 2012;98:438-42.

12. Gulati A, Jabbour A, Ismail TF, Guha K, Khwaja J, Raza S, Morarji K, Brown TD, Ismail NA, Dweck MR, et al. Association of fibrosis with mortality and sudden cardiac death in patients with nonischemic dilated cardiomyopathy. JAMA. 2013;309:896-908

13. Simonetti OP, Kim RJ, Fieno DS, Hillenbrand HB, Wu E, Bundy JM, Finn JP, Judd RM. An improved MR imaging technique for the visualization of myocardial infarction. Radiology. 2001;218:215-23.

14. Kim RJ, Wu E, Rafael A, Chen EL, Parker MA, Simonetti O, Klocke FJ, Bonow $\mathrm{RO}$, Judd RM. The use of contrast-enhanced magnetic resonance imaging to identify reversible myocardial dysfunction. N Engl J Med. 2000;343:1445-53.

15. Rutz T, Piccini D, Coppo S, Chaptinel J, Ginami G, Vincenti G, Stuber M, Schwitter J. Improved border sharpness of post-infarct scar by a novel selfnavigated free-breathing high-resolution 3D whole-heart inversion recovery magnetic resonance approach. Int J Cardiovasc Imaging. 2016;32:1735-44.

16. Kellman $\mathrm{P}$, Arai $\mathrm{AE}, \mathrm{MCV}$ eigh $\mathrm{ER}$, Aletras $\mathrm{AH}$. Phase-sensitive inversion recovery for detecting myocardial infarction using gadolinium-delayed hyperenhancement. Magn Reson Med. 2002;47:372-83.

17. Yamashita S, Sacher F, Mahida S, Berte B, Lim HS, Komatsu Y, Amraoui S, Denis A, Derval N, Laurent F, et al. Image integration to guide catheter ablation in scar-related ventricular tachycardia. J Cardiovasc Electrophysiol. 2016;27:699-708

18. Kurzendorfer T, Forman C, Schmidt M, Tillmanns C, Maier A, Brost A. Fully automatic segmentation of left ventricular anatomy in 3-D LGE-MRI. Comput Med Imaging Graph. 2017;59:13-27.

19. Kellman P, Xue H, Olivieri $\sqcup$, Cross RR, Grant EK, Fontana M, Ugander M, Moon JC, Hansen MS. Dark blood late enhancement imaging. J CardiovasC Magn Reson. 2016;18:77.

20. Liu CY, Wieben O, Brittain JH, Reeder SB. Improved delayed enhanced myocardial imaging with T2-prep inversion recovery magnetization preparation. J Magn Reson Imaging. 2008;28:1280-6.

21. Basha TA, Tang MC, Tsao C, Tschabrunn CM, Anter E, Manning WJ, Nezafat R. Improved dark blood late gadolinium enhancement (DB-LGE) imaging using an optimized jointinversion preparation and T2 magnetization preparation. Magn Reson Med. 2017. doi:10.1002/mrm.26692. [Epub ahead of print].

22. Kino A, Zuehlsdorff S, Sheehan JJ, Weale PJ, Carroll TJ, Jerecic R, Carr JC. Three-dimensional phase-sensitive inversion-recovery turbo FLASH sequence for the evaluation of left ventricular myocardial scar. AJR Am J Roentgenol. 2009:193:W381-8.

23. Kido T, Kido T, Nakamura M, Kawaguchi N, Nishiyama Y, Ogimoto A, Miyagawa M, Mochizuki T. Three-dimensional phase-sensitive inversion recovery sequencing in the evaluation of left ventricular myocardial scars in ischemic and non-ischemic cardiomyopathy: comparison to threedimensional inversion recovery sequencing. Eur J Radiol. 2014;83:2159-66.

24. Ehman RL, Felmlee JP. Adaptive technique for high-definition MR imaging of moving structures. Radiology. 1989;173:255-63.

25. Keegan J, Drivas P, Firmin DN. Navigator artifact reduction in threedimensional late gadolinium enhancement imaging of the atria. Magn Reson Med. 2014;72:779-85.

26. Ginami G, Neji R, Phinikaridou A, Whitaker J, Botnar RM, Prieto C. Simultaneous bright- and black-blood whole-heart MRI for noncontrast enhanced coronary lumen and thrombus visualization. Magn Reson Med. 2017. doi:10.1002/mrm.26815. [Epub ahead of print].

27. Henningsson M, Koken P, Stehning C, Razavi R, Prieto C, Botnar RM. Wholeheart coronary MR angiography with $2 \mathrm{D}$ self-navigated image reconstruction. Magn Reson Med. 2012;67:437-45.

28. Prieto C, Doneva M, Usman M, Henningsson M, Greil G, Schaeffter T, Botnar RM. Highly efficient respiratory motion compensated free-breathing coronary MRA using golden-step Cartesian acquisition. J Magn Reson Imaging. 2015;41:738-46

29. Sussman MS, Wright GA. Factors affecting the correlation coefficient template matching algorithm with application to real-time 2-D coronary artery MR imaging. IEEE Trans Med Imaging. 2003;22:206-16.

30. Piccini D, Bonanno G, Ginami G, Littmann A, Zenge MO, Stuber M. Is there an optimal respiratory reference position for self-navigated whole-heart coronary MR angiography? J Magn Reson Imaging. 2016;43:426-33.

31. Weigel M. Extended phase graphs: dephasing, RF pulses, and echoes - pure and simple. J Magn Reson Imaging. 2015;41:266-95.
32. Kim WY, Danias PG, Stuber M, Flamm SD, Plein S, Nagel E, Langerak SE, Weber OM, Pedersen EM, Schmidt M, et al. Coronary magnetic resonance angiography for the detection of coronary stenoses. N Engl J Med. 2001; 345:1863-9.

33. Captur G, Gatehouse P, Keenan KE, Heslinga FG, Bruehl R, Prothmann M, Graves MJ, Eames RJ, Torlasco C, Benedetti G, et al. A medical device-grade $\mathrm{T} 1$ and ECV phantom for global T1 mapping quality assurance-the T1 mapping and ECV standardization in cardiovascular magnetic resonance (T1MES) program. J Cardiovasc Magn Reson. 2016;18:58.

34. Messroghli DR, Walters K, Plein S, Sparrow P, Friedrich MG, Ridgway JP, Sivananthan MU. Myocardial T1 mapping: application to patients with acute and chronic myocardial infarction. Magn Reson Med. 2007;58:34-40.

35. De Wilde JP, Lunt JA, Straughan K. Information in magnetic resonance images: evaluation of signal, noise and contrast. Med Biol Eng Comput. 1997:35:259-65.

36. Griswold MA, Jakob PM, Heidemann RM, Nittka M, Jellus V, Wang J, Kiefer B, Haase A. Generalized autocalibrating partially parallel acquisitions (GRAPPA). Magn Reson Med. 2002;47:1202-10.

37. Wang $Y$, Riederer SJ, Ehman RL. Respiratory motion of the heart: kinematics and the implications for the spatial resolution in coronary imaging. Magn Reson Med. 1995;33:713-9.

38. Amano $\mathrm{Y}$, Onda $\mathrm{M}$, Amano $\mathrm{M}$, Kumazaki T. Magnetic resonance imaging of myelofibrosis. STIR and gadolinium-enhanced MR images. Clin Imaging. 1997;21:264-8.

39. Haase A, Frahm J, Hanicke W, Matthaei D. 1H NMR chemical shift selective (CHESS) imaging. Phys Med Biol. 1985;30:341-4.

40. Amano Y, Kiriyama T, Kobayashi Y, Tachi M, Matsumura Y, Kumita S. Simultaneous assessment of myocardial scar and coronary arteries using navigator-gated 3-dimensional fat-suppressed delayed-enhancement MRI at 3.0 T: a technical feasibility study. J Comput Assist Tomogr. 2012;36:72-6.

41. Sodickson DK, Manning WJ. Simultaneous acquisition of spatial harmonics (SMASH): fast imaging with radiofrequency coil arrays. Magn Reson Med. 1997:38:591-603.

42. Lustig M, Donoho D, Pauly JM. Sparse MRI: the application of compressed sensing for rapid MR imaging. Magn Reson Med. 2007:58:1182-95.

43. Manke D, Nehrke K, Bornert $P$, Rosch P, Dossel O. Respiratory motion in coronary magnetic resonance angiography: a comparison of different motion models. J Magn Reson Imaging. 2002;15:661-71.

44. Manke D, Nehrke K, Bornert P. Novel prospective respiratory motion correction approach for free-breathing coronary MR angiography using a patient-adapted affine motion model. Magn Reson Med. 2003;50:122-31.

45. Shechter G, Ozturk C, Resar JR, McVeigh ER. Respiratory motion of the heart from free breathing coronary angiograms. IEEE Trans Med Imaging. 2004;23: 1046-56.

46. Cruz G, Atkinson D, Henningsson M, Botnar RM, Prieto C. Highly efficient nonrigid motion-corrected 3D whole-heart coronary vessel wall imaging. Magn Reson Med. 2016; 10.1002/mrm.26274.

47. Ginami G, Bonanno G, Schwitter J, Stuber M, Piccini D. An iterative approach to respiratory self-navigated whole-heart coronary MRA significantly improves image quality in a preliminary patient study. Magn Reson Med. 2016:75:1594-604

\section{Submit your next manuscript to BioMed Central and we will help you at every step:}

- We accept pre-submission inquiries

- Our selector tool helps you to find the most relevant journal

- We provide round the clock customer support

- Convenient online submission

- Thorough peer review

- Inclusion in PubMed and all major indexing services

- Maximum visibility for your research

Submit your manuscript at www.biomedcentral.com/submit 